\title{
GEANT4 simulation of optical modules in neutrino telescopes
}

\author{
Christophe M.F. Hugon* \\ INFN Sezione di Genova \\ E-mail: christophe.hugonege.infn.it
}

\begin{abstract}
Neutrinos have a very important role in the multi-messenger astronomy, therefore, in recent years, larges underwater and under-ice neutrinos telescopes have been designed to allow the detection of high energy neutrinos. The neutrino energy spectrum and direction are inferred based on the detection of the Cherenkov light induced by the secondary charged particles in the medium. Optical modules (OMs), i.e. glass spheres containing the photomultiplier tubes (PMTs) for light detection at the single photon level, and the associated electronics, are central components of such neutrino telescopes. They require detailed simulations which are usually based on parametrized results of laboratory measurements. Here an alternative approach is presented, where the light detection in OMs is simulated using the latest GEANT4 simulation library. This simulation reproduces faithfully the experimental conditions, easily allowing the definition of any geometry of the OMs and common PMT types to simulate precisely the photon propagation in various detection units geometry. A precise simulation of the photon interaction in the photocathode is performed, taking into account the optical properties of bialkali photocathodes and using a dedicated algorithm. This simulation has been applied to 10-inch Hamamatsu PMTs in 17-inch spheres (ANTARES), 10-inch Hamamatsu PMTs in 13-inch spheres (NEMO), and multiple 3-inch PMTs in 17-inch spheres (KM3NeT). The methods, the results achieved and their relevance for data analysis in neutrino telescope will be presented.
\end{abstract}

The 34th International Cosmic Ray Conference,

30 July- 6 August, 2015

The Hague, The Netherlands

* Speaker. 


\section{The neutrino telescopes ANTARES NEMO and KMBNeT}

Among the messengers to observe the universe, the neutrino is a privileged particle, due to its properties. Indeed, its very low cross section and its null charge allow it to emerge from dense media and to travel straight undeflected through very long distances. Neutrinos at high energies $(>\mathrm{TeV})$ are expected to be emitted by a large range of astrophysical objects (supernovae, GRB etc.), and potentially from unexpected sources or processes.

The Neutrino telescopes require a very large effective volume of a transparent material such as water or ice. Thanks to an array of photo-detectors, the Cherenkov light emitted by the charged lepton (produced by electroweak interaction between the neutrino and the matter) can be detected. The detection and reconstruction of the Cherenkov ligth cone by photo-detectors units permits to determine the direction of the parent neutrino and an estimation of its energy. The earth represents a screen to charged secondary cosmic rays, so the detectors are installed in deep depth (to limit the down-going background) and are optimized to measure the up-going muons, generated by neutrino traversing the Earth.

This section will describe the neutrino telescopes to which the GEANT4 simulation presented in the further sections has been applied.

ANTARES [1] is currently the largest deep-sea neutrino telescope in the Northern Hemisphere. It is situated in front of Toulon (France) at about $2500 \mathrm{~m}$ depth in the Mediterranean Sea. The apparatus is made up of 12 lines of $450 \mathrm{~m}$ high. These lines are separated by a space from 60 to $75 \mathrm{~m}$. Each line support 25 storeys of Optical Modules (OMs) triplet. The OMs contain 10" Hamamatsu R7081-20 Photo-Multiplier tubes (PMTs), enclosed in 17" pressure-proof glass spheres and are oriented $45^{\circ}$ downward.

The NEMO prototype [2], was deployed in 2013 to validate the $3500 \mathrm{~m}$ depth site near CapoPassero (Sicily) for a $\mathrm{km}^{3}$ scale detector. This prototype apparatus consists of a semi-rigid structure composed of 8 floors, which consists of an $8 \mathrm{~m}$ long metal bars, vertically spaced $40 \mathrm{~m}$ from each other. Each extremity of the bars support two OMs containing the same 10" PMTs as ANTARES, enclosed in 13" pressure-proof glass spheres.

KM3NeT [3] represents the next generation of Mediterranean neutrino telescopes. It will be deployed on three different sites : KM3NeT-Fr in front of Toulon, France; KM3NeT-It in front of Capo-Passero, Sicily, Italy; KM3NeT-Gr in front of Pilos, Peloponnese, Greece (KM3NeT$\mathrm{Gr}$ ). The apparatus is composed of building block containing 115 detections units (lines). These detection units will be separated by tens of meters (still under study). Each site will hold 2 building block to have a total fiducial volume of $1 \mathrm{~km}^{3}$. The detection units are Digital Optical Module (DOM), made up of 31 3" PMTs enclosed in the same vessel as ANTARES, i.e. 17" pressure-proof glass spheres. Moreover the first prototypes of the future KM3NeT neutrino telescope have been integrated in the instrumented line of the ANTARES detector for in-situ testing and validation [3]. A second prototype was deployed at the Capo-Passero site [3]. 


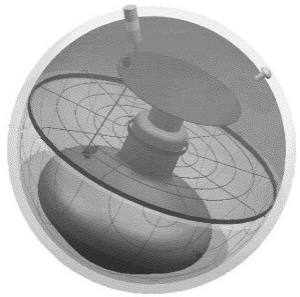

(a) Scheme of an ANTARES OM

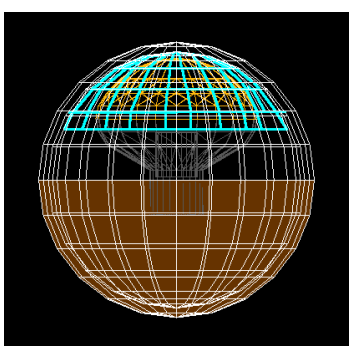

(d) Simulation of the ANTARES OM.

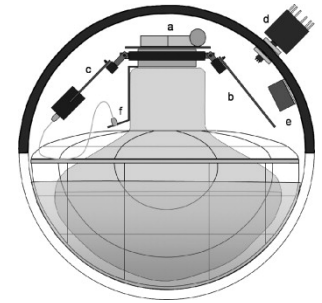

(b) Scheme of a NEMO OM

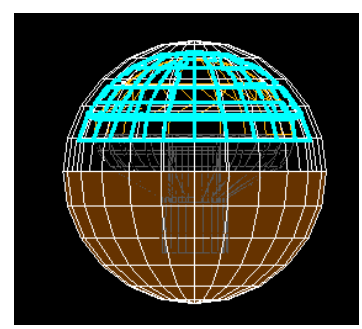

(e) Simulation of the NEMO OM.

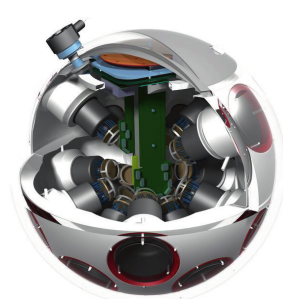

(c) Scheme of a KM3NeT DOM

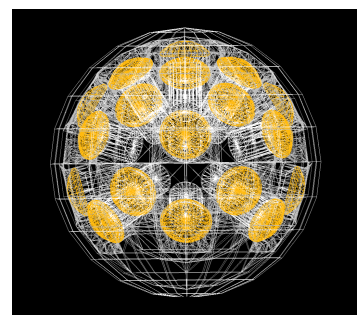

(f) Simulation of the KM3NeT DOM.

Figure 1: Illustration of OMs for ANTARES (left), NEMO (center) and a KM3NeT DOM (right), schematics (top) and simulated geometry (bottom). The colors correspond to the glass sphere (white), the gel (cyan) and the absorber (brown) and the PMTs (gray and yellow).

\section{Objectives of the ray tracing simulation}

The ray-tracing simulation is the very first step of the full simulation of ANTARES, NEMO and KM3NeT. It manages the propagation of each photon, from the Cherenkov effect or other sources, and their different interactions in the matter. When these photons reach the detection surface, the photocathode, a complementary accurate calculation process presented in section 4 is used in order to obtain a precise detection probability.

The ray tracing simulation provides the mean effects as the detection probability, the water properties etc. that are used in the further steps of the full simulation process. The goal is to provide two basics elements called:

- The OM and DOM detection efficiency as a function of the angle and the wavelength of the incident photon (angular acceptance),

- Complementary information on the water properties such as the absorptions length and the scattering length on molecule and on macroscopic particles.

These results are then tabulated and used as inputs for the next simulation step, called km3net [4].

\section{The geometry definition of the detection units}

Particular attention was given to the ray-tracing simulation geometry definition to minimize possible systematic errors induced by difference with the real geometry. Every geometrical parameter and material properties (such as the glass thickness, the index and absorption length, the material etc.) can be easily implemented and modified by the end-user thanks to a set of "easy 


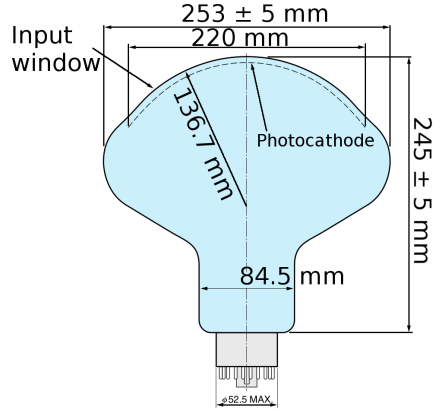

(a) R7081-20

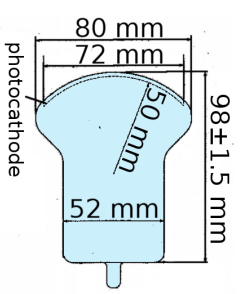

(b) R12199

Figure 2: Schemes of the R7081-20 (ANTARES and NEMO) and R12199 (KM3NeT) PMTs.

to use" configuration files. Consequently, the same software code manage the three geometries (NEMO, ANTARES and KM3NeT). The figure 1 gives a global view of the differences between the three geometries.

The noticeable components are:

- The vessel, mainly composed by the pressure proof glass, the plastic PMT support and the optical gel. The structure can also be included if needed, as for the NEMO case. Those are passive materials and the inputs are only the material properties

- The PMTs. It's geometry is more complex, and the physics used for the photo detection is based on a simulation of the photocathode, described in the section 4 .

\subsection{The photo-multiplier}

The presented ray tracing simulation is able to integrate an user defined PMT geometry thanks to the configuration files considering the basic geometrical components, which are represented in figure 2. The required parameters, provided by the manufacturers, are a detailed geometry of the glass, photocathode and inner tube (the multiplier structure) and the characteristics of the materials. The other parameters are automatically constrained by the software.

Two different types of PMT are used in this simulation:

- the Hamamatsu R7081-20 10" PMT: only one per OM, used in ANTARES and NEMO,

- the Hamamatsu T12199 3" PMTs: 31 per OM, used in KM3NeT.

\section{The photocathode physics}

For a detailed simulation also the photocathode thickness and refractive index are fundamental parameters. The ANTARES, NEMO and KM3NeT PMTs photocathodes are a bialkali one [5] and the thickness and refractive index have been obtained by a dedicated measurement reported in [6]. In the commonly used simplified model, the detection is a function of the probability that an optical photon is absorbed by the photocathode and that is converted to a photo-electron. This is known as the PMT quantum efficiency, is dependent on the photon wavelength and is experimentally measured and provided by HAMAMATSU.

In the general case of a PMT in air, this approximation is sufficient because the incident angle aperture of the photon in respect to the photocathode is limited due to the high index difference be- 

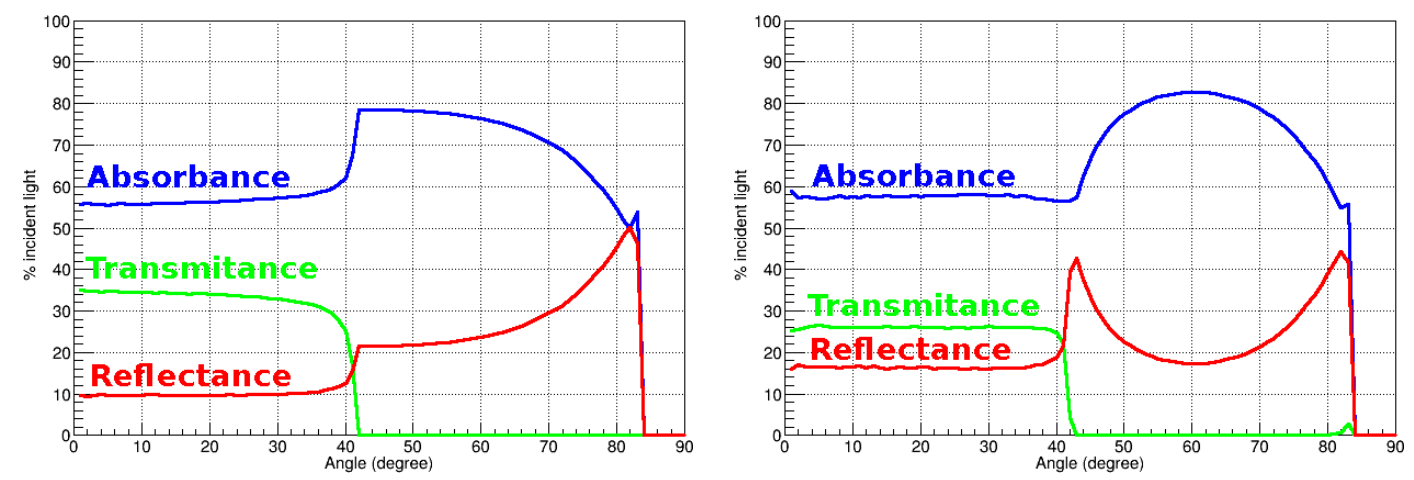

(a) Simulation results with the classic GEANT4 optics.

(b) Simulation results with the thin layer extension in GEANT4 optics, in accordance to the results in [8].

Figure 3: Simulations of photons interaction with a bialkali photocathode, assuming the following parametrization: $\mathrm{n} 1=1.5 \mathrm{n} 1=1.5$ (external medium), $\mathrm{n} 2=1.51 \mathrm{n} 2=1.51$ (glass), $\mathrm{n} 3=2.7+1.5 \mathrm{i}$ (photocathode), $\mathrm{n} 4=1$ (vacuum); photocathode thickness $=20 \mathrm{~nm} ; \lambda=442 \mathrm{~nm}$.

tween the glass and the air. On the contrary case of an OM like the one used in neutrino telescopes, i.e. a succession of close index (water, glass, gel, glass, photocathode) allows large angles, which implies important divergences with respect to the simplified model. This divergence is basically due to:

- the thickness of the photocathode (tens of nm) that implies a complex wave construction and destruction of the photon, not managed by ray-tracing simulations,

- the absorption optics mechanism of the photocathode, based on a non null complex value of its index.

The mechanism of reflections between the photocathode and the inner vacuum is modified and does not follow the classical optics. In a more complete definition of the photocathode, both the quantum efficiency and absorbance depend on the photon wavelength $\lambda$ and the incident angle, with respect to the normal direction of the photocathode glass window, $\theta$ are taken in account.

In the work by Motta and Schönert a detailed optical model for thin layers is presented that well describes the experimental measurements of absorption, reflection and refraction probabilities in photocathodes layers $[7,8]$ that allow to deduce the complex index of the photocathode.

Therefore, a part of the code used by the DoubleChooz experiment (DCGLG4sim) [9] has been improved and implemented in the presented simulation. The figure 3 shows the results of the classical GEANT4 simulation and the new simulation thin layer in comparaison with the theoretical results reported in [8]. This thin layer model has been integrated as an extension of the GEANT4 optical boundary and is activated on interface containing properties such as a complex refractive index and a thin layer thickness.

\section{Calibration}

Once the geometry and the physics are well defined, we have obtained the number of photoelectrons produced on the internal surface of the photocathode as a function of the impact angles 
of the photon and its wavelength. The total detection efficiency, however, is still not exact, mainly due to the non-simulated components such as the glass/photocathode systematic irregularities, the collection efficiency or the geometry definition uncertainties.

In order to calibrate the OM efficiency, two important physical inputs are used: The relative angular efficiency presented in 5.1 and the absolute efficiency presented in 5.2.

\subsection{The laboratory calibration setup and the relative angular efficiency}

In the laboratory, both calibrations (angular and absolute efficiency calibrations) have been done with the same setup.

The calibration was done at ECAP (Erlangen) and at APC (Paris) [10] and was performed in a black box which has been designed in order to contain an OM, a calibrated light source (a light emitting diode of $\lambda=378 \mathrm{~nm}$ ) and motorized arms. These arms allow the movement of the light source around the tested OM or PMT.

For the relative angular measurement, the arm goes around the tested OM or PMT and allows an orthogonal efficiency scan (a total number of 18 different scans has been done).

Therefore, to perform this calibration, the OM has been simulated in the same conditions as the experimental measurements (in terms of wavelength, angles, environments...). The obtained difference between the experiment and the simulation is used to evaluate the correction factor for each angle, where the angle is defined considering the impact position of the photon in respect to the central axis of the OM and PMT.

The figure 4a represents the result of the simulated scans in comparison with the data. The black line indicates the mean values obtained with simulation for each scanned angle, the green line the mean values plus the standard deviation and the red line, the mean values minus the standard deviation obtained from simulation. The experimental mean values (blue points) are also reported for comparison.

The standard deviation is dominated by the differences of angular efficiencies between the measured OMs. The simulation standard deviation estimation is calibrated to correspond to the experimental one.

This setup allows to do an absolute efficiency measurement that, as described in section 5.2, is compatible with the in-situ ${ }^{40} \mathrm{~K}$ calibration.

In the case of KM3NeT, a direct measurement of the bare R12199 PMT was performed at ECAP and is presented in figure 4b. The size of the PMT and the dynods diameter reduce significantly the angular efficiency, which, in this case, is considered as quasi constant.

\subsection{The absolute calibration}

The absolute efficiency calibration of the OM can be obtained by two experimental measurements. The first is the absolute efficiency measurement done in the laboratory using a calibrated light source, as described in section 5.1. This measurement is done only for the ANTARES OMs.

The second way to determine the absolute efficiency calibration is to use the ${ }^{40} \mathrm{~K}$ rate on site, estimated at $13,500 \mathrm{~Bq}[11]$. The coincidence rate is measured continuously by the ANTARES, NEMO detectors [12] and KM3NeT first DU citeConiglione2015591. Therefore, by performing a simulation using the experimental conditions of the sites it is possible to directly compare experimental ans simulated results and therefore to obtain an absolute calibration of the OM. 


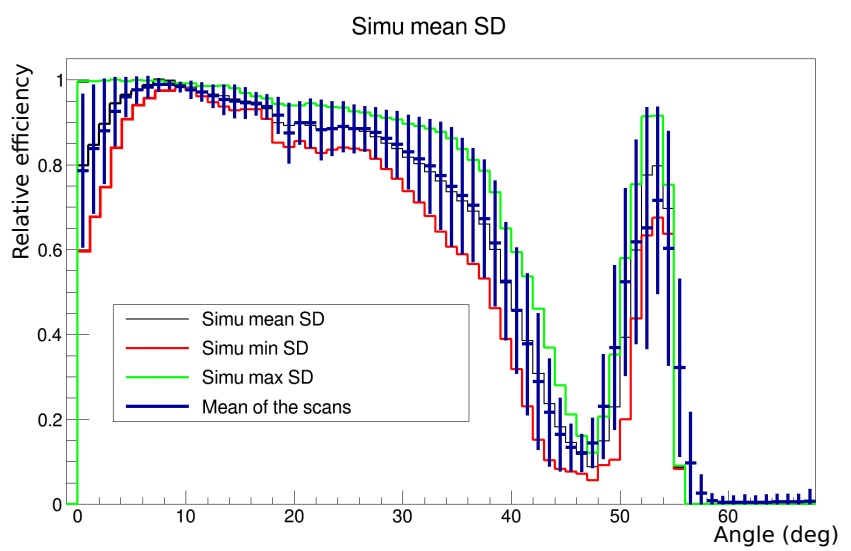

(a) Angular efficiency of the R7081-20 used in ANTARES and NEMO. The blue point are the data mean, the error bars are the standard deviation of the measurements. The black line correspond to the simulation result, the green an red lines to the estimation of the standard deviation by the simulation.

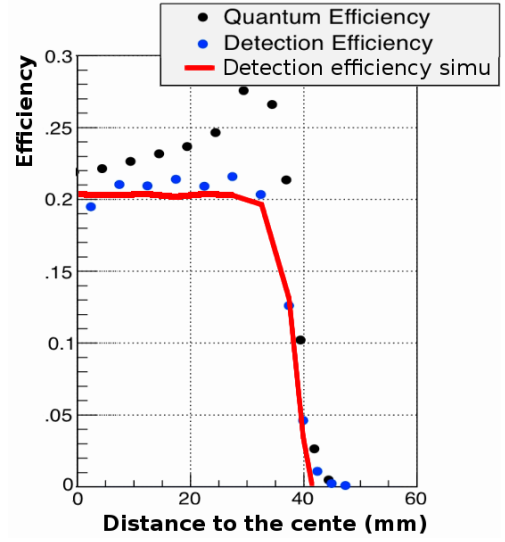

(b) Efficiency of PMT used in $\mathrm{KM} 3 \mathrm{NeT}$ in function of the distance with the center, all point beam is parallel to the PMT center normal. The red line represent the simulation in the same experimental conditions.

Figure 4: Results of the ANTARES OMs and KM3NeT PMT scans (data and simulation).

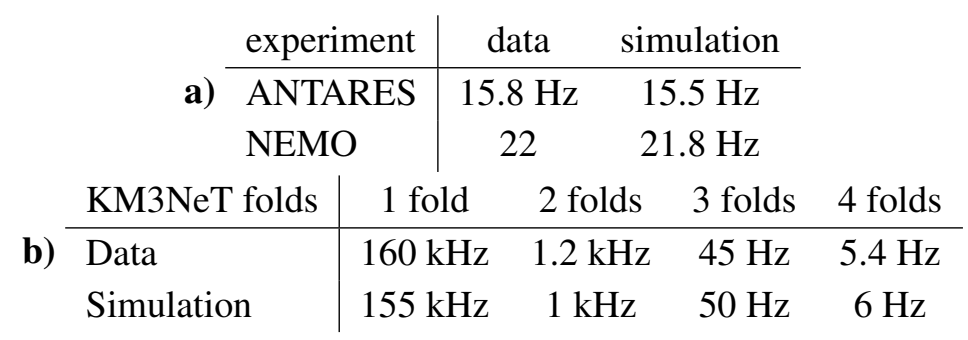

Table 1: Results of the simulation in comparison with the data for the three experiments, using a correction factor for the total efficiency of 0.81 for ANTARES and NEMO, and 0.9 for KM3NeT.

This calibration is based on the measurement of the coincidence rate between two OMs of the same floor for NEMO and ANTARES, and multiple PMTs of the same DOM for KM3NeT. These coincidences permit to exclude the bio-luminescence background and to be independent from the water properties (only close events produce a coincidence events). The experimental values for ANTARES are reported in [13] The values for NEMO are taken from a dedicated analysis and were independently cross-checked by a second analysis reported in [14].

The table 1 presents the comparison between the simulation and the data for ANTARES, NEMO experiments and the KM3NeT demonstrator. Note that by using the same correction factor, both simulations of ANTARES and NEMO ${ }^{40} \mathrm{~K}$ coincidence rate agree very well with the experimental values. Since the efficiency of the ANTARES detector drops with time, as can be noticed by the drop of the ${ }^{40} \mathrm{~K}$ coincidence rate between 2009 and 2011, a different correction factor should be obtained for ANTARES according to the different data-taking period. 
By using either the laboratory measurement or the ${ }^{40} \mathrm{~K}$ coincidence rate, the same correction factor of 0.81 has been obtained for the NEMO and ANTARES OM. The correction factor for the 3" PMT is smaller, about 0.9 . Those correction factors correspond to the collection efficiency. This result gives confidence on the reliability of the OM simulation.

\section{Conclusion and perspectives}

Several new improvements to the ray-tracing simulation, such as the geometry refinement, the wavelength and angular dependency of the efficiency and-the relative and absolute calibration methodologies are reported. The current simulation uses well understood processes, and it is now considered very accurate and already brought numerous applications for neutrino detectors as ANTARES, NEMO or the future KM3NeT such as the angular acceptance estimation, the water properties estimations. The ${ }^{40} \mathrm{~K}$ decay rate and the water properties measurements have validated the software.

\section{References}

[1] V. Van Elewyck. Recent results from the ANTARES neutrino telescope. NIMA, 742(0):63 - 70, 2014.

[2] C.A. Nicolau. From NEMO to km3net-italy. NIM A, 742(0):203 - 207, 2014.

[3] R. Coniglione. The status of the KM3NeT Project. Physics Procedia, 61(0):591 - 597, 2015. 13th International Conference on Topics in Astroparticle and Underground Physics, TAUP 2013.

[4] C. James. Internal note: km3net release. 2014.

[5] ANTARES coll. The ANTARES optical module. NIM A, 484(1-3):369 - 383, 2002.

[6] M.E. Moorhead and N.W. Tanner. Optical properties of an $\{\mathrm{EMI}\}\{\mathrm{K} 2 \mathrm{CsSb}\}$ bialkali photocathode. NIM A, 378(1-2):162 - 170, 1996.

[7] A. Creusot and D. Veberič. Simulation of large photomultipliers for experiments in astroparticle physics. NIM A, 613(1):145 - 151, 2010.

[8] D. Motta and S. Sch Optical properties of bialkali photocathodes. NIMA, 539(1?2):217 - 235, 2005.

[9] D. Motta. Glg4 simulation.

[10] A. Creusot and al. PMT measurements in ANTARES. NIM A, 725(0):144 - 147, 2013. VLVvT 11, Erlangen, Germany, 12 - 14 October, 2011 5th International Workshop on Very Large Volume Neutrino Telescopes, The future of high-energy neutrino astronomy.

[11] ANTARES coll. Background light in potential sites for the ANTARES undersea neutrino telescope. Astroparticle Physics, 13(2âĂŞ3):127 - 136, 2000.

[12] G. Riccobene et al. Deep seawater inherent optical properties in the southern ionian sea. Astroparticle Physics, 27(1):1 - 9, 2007.

[13] ANTARES coll. Time calibration of the ANTARES neutrino telescope. Astroparticle Physics, 34(7):539 - 549, 2011.

[14] P. Fermani. Internal note, first estimation rate of the $\mathrm{k} 40$ coincidences in the nemo phase 2 experiment. 2014. 